\author{
Marquette University \\ e-Publications@Marquette
}

Electrical and Computer Engineering Faculty

Research and Publications

Electrical and Computer Engineering,

Department of

\title{
SAR-Based Vibration Estimation Using the Discrete Fractional Fourier Transform
}

\author{
Qi Wang \\ University of New Mexico - Albuquerque \\ Matthew Pepin \\ University of New Mexico \\ Ryan J. Beach \\ University of New Mexico \\ Ralf Dunkel \\ General Atomics Aeronautical Systems \\ Tom Atwood \\ Sandia National Laboratories
}

See next page for additional authors

Follow this and additional works at: https://epublications.marquette.edu/electric_fac

Part of the Computer Engineering Commons, and the Electrical and Computer Engineering Commons

\section{Recommended Citation}

Wang, Qi; Pepin, Matthew; Beach, Ryan J.; Dunkel, Ralf; Atwood, Tom; Santhanam, Balu; Gerstle, Walter; Doerry, Armin W.; and Hayat, Majeed M., "SAR-Based Vibration Estimation Using the Discrete Fractional Fourier Transform" (2012). Electrical and Computer Engineering Faculty Research and Publications. 571. https://epublications.marquette.edu/electric_fac/571 
Authors

Qi Wang, Matthew Pepin, Ryan J. Beach, Ralf Dunkel, Tom Atwood, Balu Santhanam, Walter Gerstle, Armin W. Doerry, and Majeed M. Hayat

This article is available at e-Publications@Marquette: https://epublications.marquette.edu/electric_fac/571 
Marquette University

e-Publications@Marquette

\section{Electrical and Computer Engineering Faculty Research and Publications/College of Engineering}

This paper is NOT THE PUBLISHED VERSION; but the author's final, peer-reviewed manuscript. The published version may be accessed by following the link in the citation below.

IEEE Transactions on Geoscience and Remote Sensing, Vol. 50, No. 10 (October 2012): 4145-4156. DOI. This article is (C) Institute of Electrical and Electronic Engineers (IEEE) and permission has been granted for this version to appear in e-Publications@Marquette. Institute of Electrical and Electronic Engineers (IEEE) does not grant permission for this article to be further copied/distributed or hosted elsewhere without the express permission from Institute of Electrical and Electronic Engineers (IEEE).

\section{SAR-Based Vibration Estimation Using the Discrete Fractional Fourier Transform}

\section{Qi Wang}

Center for High Technology Materials and the Department of Electrical and Computer Engineering, University of New Mexico, Albuquerque, NM

Matthew Pepin

Center for High Technology Materials and the Department of Electrical and Computer Engineering, University of New Mexico, Albuquerque, NM

Ryan J. Beach

Departments of Electrical and Computer Engineering and Mechanical Engineering, University of New Mexico, Albuquerque, NM

Ralf Dunkel

General Atomics Aeronautical Systems, Inc., San Diego, CA

Tom Atwood

Sandia National Laboratories, Albuquerque, NM

Balu Santhanam 
Departments of Electrical and Computer Engineering and Mechanical Engineering, University of New Mexico, Albuquerque, NM

\title{
Walter Gerstle
}

Department of Civil Engineering, University of New Mexico, Albuquerque, NM

\section{Armin W. Doerry}

Sandia National Laboratories, Albuquerque, NM

\section{Majeed M. Hayat}

Center for High Technology Materials and the Department of Electrical and Computer Engineering, University of New Mexico, Albuquerque, NM

\begin{abstract}
:
A vibration estimation method for synthetic aperture radar (SAR) is presented based on a novel application of the discrete fractional Fourier transform (DFRFT). Small vibrations of ground targets introduce phase modulation in the SAR returned signals. With standard preprocessing of the returned signals, followed by the application of the DFRFT, the time-varying accelerations, frequencies, and displacements associated with vibrating objects can be extracted by successively estimating the quasi-instantaneous chirp rate in the phase-modulated signal in each subaperture. The performance of the proposed method is investigated quantitatively, and the measurable vibration frequencies and displacements are determined. Simulation results show that the proposed method can successfully estimate a two-component vibration at practical signal-to-noise levels. Two airborne experiments were also conducted using the Lynx SAR system in conjunction with vibrating ground test targets. The experiments demonstrated the correct estimation of a 1- $\mathrm{Hz}$ vibration with an amplitude of $1.5 \mathrm{~cm}$ and a 5$\mathrm{Hz}$ vibration with an amplitude of $1.5 \mathrm{~mm}$.
\end{abstract}

\section{Keywords}

Vibrations, Chirp, History, Estimation, Discrete Fourier transforms, Acceleration, Signal to noise ratio

\section{SECTION I. Introduction}

Vibration signatures associated with objects such as active structures (e.g., bridges and buildings) and vehicles can bear vital information about the type and integrity of these objects. The ability to remotely sense minute structural vibrations persistently and with high accuracy is extremely important for a number of reasons. First, it avoids the cost of acquiring and installing accelerometers on remote structures. Second, it alleviates the high cost of maintaining these sensors, and third, it enables sensing vibrations of structures that are not easily accessible to engineers and maintenance personnel (e.g., pedestrian and train bridges over canyons, structures and vehicles in a hostile land, etc.). While light detection and ranging (LIDAR) technology has been proposed and used for remote sensing of vibrations, it has failed to overcome a number of persisting challenges. First, due to the short wavelength of the standard illumination in LIDAR, loss and aberration due to laser propagation through air and vapor make LIDAR vibration sensing highly dependent on weather conditions. This makes it particularly problematic when it is desirable to probe vibrating object at a large distance (tens of kilometers or more). Second, LIDAR systems are not typically easily mounted on small moving platforms due to the complexity of the system.

Synthetic aperture radar (SAR) is a well-established technique for high-resolution imaging of the Earth's surface through measurement of its electromagnetic reflectivity [1]-[2][3]. The relatively long wavelengths, compared with those of optical sensors, make SAR systems capable of remote imaging over thousands of kilometers 
regardless of weather conditions. In addition, small vibrations in the imaged surfaces introduce phase modulation in the reflected SAR signals, a phenomenon often referred to as the micro-Doppler effect [4][5][6][7][8][9]. As such, in addition to imaging, SAR can also have the added benefit of enabling us to remotely measure surface vibrations by estimating the corresponding micro-Doppler effect.

In standard SAR imaging, vibrations from strong scatterers result in "ghost targets" around the scatterers in the SAR image (in the azimuth direction) that are generally difficult to distinguish from images of static scatterers [10]. This ghosting is due to the fact that the returned SAR signals, even after they are preprocessed (range compressed and autofocused), still bear the vibration-induced time-varying phase, and the standard Fourier-transform-based analysis used in SAR processing is inadequate to resolve such nonstationary signals. Indeed, the SAR returned echo from a vibrating scatterer after preprocessing is a nonstationary signal whose instantaneous frequency (IF) is linearly proportional to the vibration velocity [11]. To address this limitation of standard SAR, joint time-frequency analysis (JTFA) has been proposed to analyze the micro-Doppler effect [4]. Different time-frequency transforms have been used, including the short-time Fourier transform [12], Cohen's class transform [13], and the adaptive time-frequency transform [14]. More comprehensive reviews on timefrequency methods are available in the literature; see, for example, [4]. Nonetheless, the JTFA merely provides a qualitative illustration of the vibration-induced frequency modulation in the time-frequency representation, and it does not provide an estimation of the vibration amplitude and frequency. Additional estimation procedures, such as retrieving the IF track from the time-frequency representation, are needed in order to estimate the vibration. This step is not trivial when the signal-to-noise ratio (SNR) is low. Moreover, because the existing JTFA stops at the analysis stage, the capability and performance of the SAR-based vibration estimation are left uninvestigated.

In this paper, a vibration estimation method using SAR is presented based on a novel application of the discrete fractional Fourier transform (DFRFT). The proposed method provides a complete estimation of the vibration signature by offering the history of the instantaneous acceleration and the spectrum of the vibrating object. In this method, the conventional SAR processing procedure is performed to obtain a nonstationary signal from the vibrating target. First, the returned SAR signals are demodulated, and the polar-to-rectangular resampling is applied to the SAR phase history to correct the range cell migration. Second, "autofocus" is performed, and range compression is applied to the reformatted SAR phase history. Next, the signal from a vibrating target is focused on a range line, and it is the aforementioned nonstationary signal. After the preprocessing, the nonstationary signal is approximated by a chirp signal in a small time window, called the subaperture. The DFRFT is then applied to estimate the vibration acceleration in sliding subapertures. The performance of the proposed method is quantified in terms of the measurable frequencies and displacements, and the efficacy of the approach is demonstrated by experiments using the Lynx SAR system built by General Atomics Aeronautical Systems, Inc. (GA-ASI) [15].

The remainder of this paper is organized as follows. In Section II, we provide a theoretical analysis of the vibration-induced frequency modulation. In Section III, the DFRFT-based vibration estimation method is introduced, follow by performance analysis in Section IV. Simulations and experiments are provided in Sections $\mathrm{V}$ and $\mathrm{VI}$, respectively. Section VII contains our conclusions.

\section{SECTION II. Model}

\section{A. Motion Model}

Fig. 1 shows a 3-D SAR flight geometry, with a vibrating target located at the origin. The nominal line-of-sight distance from the target to the radar sensor is $r_{0}$, with the radar sensor located at polar angles $\psi$ and $\theta$ to the 
target. Let $r_{d}(t)$ denote the projection of the vibration displacement onto the line of sight from the target to the SAR sensor; the range of the vibrating target becomes

$r(t) \approx r_{0}-r_{d}(t) \cdot(1)$

Due to the change of aspect angle of the target during the SAR data-collection process, the range $r_{0}$ changes a little. However, modern SAR compensates for the change via proper modeling and post-signal-processing technique [1]-[2][3]. The projection $r_{d}(t)$ is also modulated by the change of aspect angle. For broadside SAR, the project can be approximated by

$r_{d}(t) \approx r_{d 0} \cos \theta(t)(2)$

where $r_{d 0}$ represents the projection of the vibration displacement for $\theta=0$. The change of aspect angle $\theta(t)$ due to the SAR geometry is known; therefore, we can estimate $r_{d 0}(t)$ from $r_{d}(t)$. For spotlight-mode SAR, the change of aspect angle is usually small [3]. In this case, we have $r_{d}(t) \approx r_{d 0}(t)$. In this paper, we consider the case of broadside spotlight-mode SAR for which the aforementioned approximation is valid.

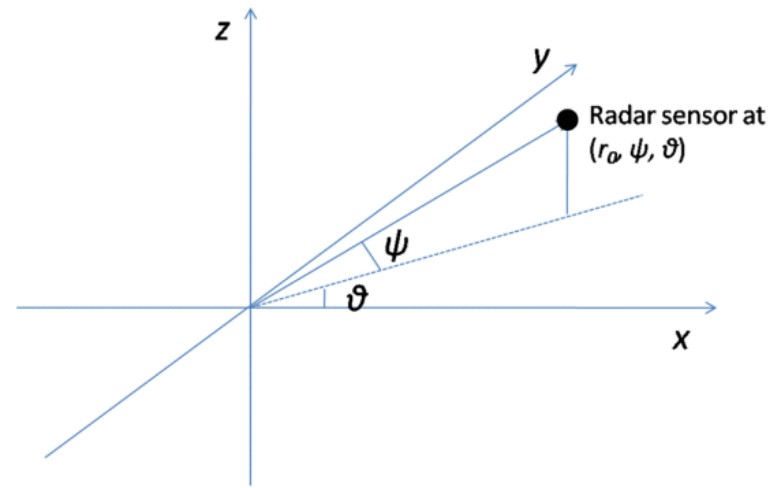

Fig. 1. Three-Dimensional SAR Flight Geometry. the Vibrating Target is Located at the Origin, and the Radar Sensor is Located at $\left(r_{0}, \psi, \theta\right)$.

\section{B. Signal Model}

The small range perturbation of the vibrating target modulates the collected SAR phase history. Consider a spotlight-mode SAR whose sent pulse is a chirp signal, with carrier frequency $f_{c}$ and chirp rate $K$. Each returned SAR pulse is demodulated by the sent pulse delayed appropriately by the round-trip time to the center of the illuminated patch. A demodulated pulse can be written as [3, Ch. 1]

$r(t)=\sum_{i} \sigma_{i} \exp \left[-j \frac{4 \pi\left(r_{i}-r_{c}\right)}{c}\left(f_{c}+K\left(t-\frac{2 r_{c}}{c}\right)\right)\right]$ (3)

where $\sigma_{i}$ is the reflectivity of the $i$ th scatterer, $c$ is the propagation speed of the pulse, and $r_{c}$ is the distance from the patch center to the antenna. The polar-to-rectangular resampling is then applied to the SAR phase history [3, Sec. 3.5] to correct for range cell migration. The autofocus is also performed at this stage. For small vibrations, the vibration-induced phase modulation in range direction is very small [4], [5], [16]; therefore, it is ignored. Range compression is applied to the phase history to separate the scatterers in range. Fig. 2 shows the magnitude of the range-compressed SAR phase history containing one static point target and one vibrating point target. Assuming that all scatterers at a specific range are static, the range-compressed phase history at this specific range can be written as

$x[n]=\sum_{i} \sigma_{i}[n] \exp \left[j\left(f_{y} y_{i} n-\frac{4 \pi f_{c}}{c} r_{i}+\phi_{i}\right)\right]+w[n](4)$ 
for $0 \leq n<N_{I}$, where $n$ is the index of the collected returned pulses, $N_{I}$ represents the total number of collected returned pulses, $y_{i}$ is the cross-range position of the $i$ th target, and $\phi_{i}$ represents all additional (constant) phase terms. The imaging factor $f_{y}$ is known and used to estimate the cross-range of the target. For spotlight-mode SAR, $f_{y}$ can be written as [2], [3]

$f_{y}=\frac{4 \pi f_{c}}{c} \frac{V}{R_{0} f_{\mathrm{prf}}}(5)$

where $V$ is the nominal speed of the SAR antenna, $R_{0}$ is the distance from the patch center to the midaperture, and $f_{\text {prf }}$ is the pulse-repetition frequency (PRF). The SAR integration time is given by $T_{I}=f_{\text {prf }} N_{I}$, and $w[n]$ is additive noise.

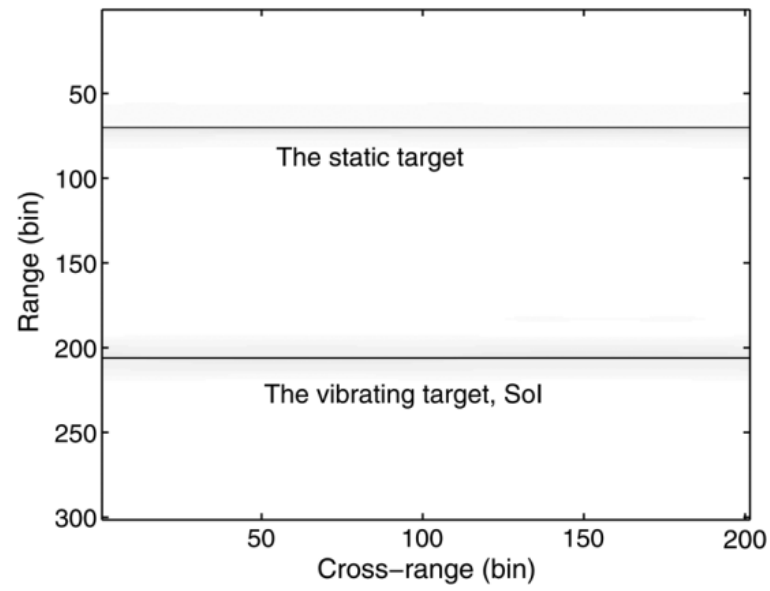

Fig. 2. Magnitude of the Range-Compressed SAR Phase History Containing one Static point Target and one Vibrating Point Target. the Two Point Targets are Separated in Range after Range Compression.

The signal $x[n]$ in $\underline{(4)}$ is a stationary signal if all scatterers are static. The azimuth compression, accomplished by applying the discrete Fourier transform (DFT) to $x[n]$, will focus the static scatterers on the correct cross-range positions. However, when a vibrating scatterer is present, $x[n]$ has a nonstationary component because $r_{i}$ is now a function of $\mathrm{n}$ for the vibrating scatterer. The cross-range $y_{i}$ is also changing for the vibrating scatterer. However, because $R_{0}$ is very large (tens of kilometers), $f_{y}$ is usually much smaller than $4 \pi f_{c} / c$; therefore, the phase modulation induced by time-varying $y_{i}$ is ignored [4], [5]. As such, we use $\bar{y}_{i}$ to denote the average crossrange position of the vibrating scatterer. For the same reason, a small change in $r_{i}$ causes a relatively large fluctuation to the Doppler frequency $f_{y} y_{i}$. We would like to emphasize that azimuth compression cannot focus the vibrating scatterer on the correct cross-range position because the DFT spectrum of the nonstationary component usually has significant sidelobes [10]. Fig. 3 shows the reconstructed SAR image by applying azimuth compression to the phase history as shown in Fig. 2. The sidelobes near the vibration target are commonly referred to as the ghost targets [10]. The vibration-induced phase modulation is referred to as the microDoppler effect [4]. Analysis tools other than the DFT are required to estimate vibrations and nonstationary targets in general. 


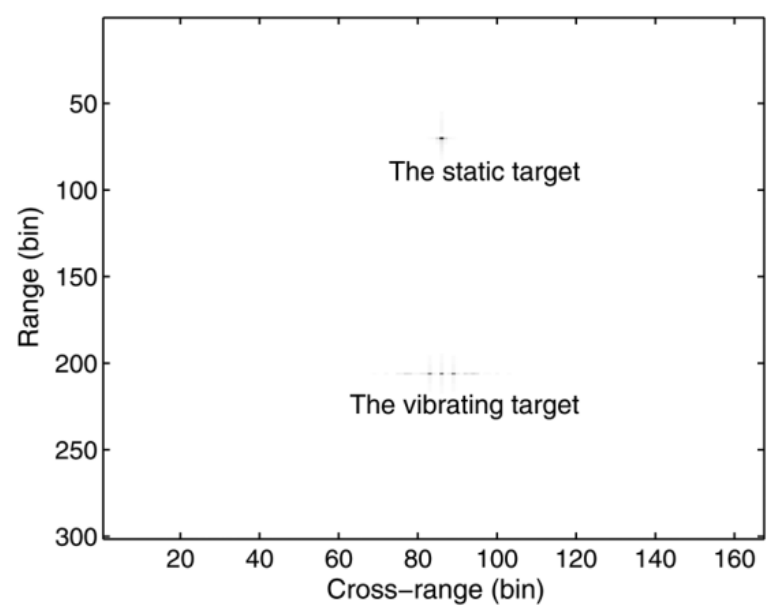

Fig. 3. Reconstructed SAR Image Using the SAR Phase History in Fig. 2. Target Vibration Introduces Ghost Targets Along the Azimuth Direction.

We define the signal of interest (Sol) as the range line in the range-compressed phase history containing vibrating targets. An example is shown in Fig. 2. In this paper, we consider cases for which the vibrating scatterer is well separated from other scatterers in range (e.g., this may be possible by choosing a proper data-collection orientation). In this case, the Sol can be written as

$x[n]=\sigma[n] \exp \left[j\left(f_{y} \bar{y} n-\frac{4 \pi f_{c}}{c} r_{d}[n]+\phi\right)\right]+w[n](6)$

for $0 \leq n<N$. In the next section, the DFRFT-based method is described and used to estimate the vibration $r_{d}[n]$ from $x[n]$.

\section{SECTION III. Algorithm Development}

For its key role in our estimation process, we will first review germane aspects of the DFRFT drawing freely from the literature [17], [18]. The vibration estimation method is then developed.

\section{A. Review of the DFRFT}

The continuous-time fractional Fourier transform, first introduced by Namias in 1980 [19], is a powerful timefrequency analysis tool for nonstationary signals and has been found to have several applications in optics and signal processing [20]. Santhanam and McClellan [21] were the first to introduce a formulation of the DFRFT. Other formulations of the DFRFT are described in the excellent review paper by Pei and Ding [22]. The DFRFT formation used in this paper is specifically referred to as the multiangle centered DFRFT (MA-CDFRFT) in the literature [17]. More details can be found in [17] and [18]. Without ambiguity, we refer to the MA-CDFRFT as the DFRFT throughout the remainder of this paper.

Let $\mathbf{W}$ denote the transformation matrix of the centered DFT. The fractional power of $\mathbf{W}$ is defined as $\mathbf{W}_{\alpha}=$ $\mathbf{V}_{G} \Lambda^{2 \alpha / \pi} \mathbf{V}_{G}^{\mathrm{T}}$, where $\mathbf{V}_{G}$ is the matrix of Grünbaum eigenvectors of $\mathbf{W}$ and $\boldsymbol{\Lambda}^{2 \alpha / \pi}$ is a diagonal matrix with the fractional powers of the eigenvalues of $\mathbf{W}$. Assume that $x[n]$ is a sequence of $N$ samples. The DFRFT of $x[n]$ is the DFT of an intermediate signal $\hat{x}_{k}[p]$ for each index $k$, i.e.,

$X_{k}[r]=\sum_{p=0}^{N-1} \hat{x}_{k}[p] \exp \left(-j \frac{2 \pi}{N} p r\right)$ (7)

where $r=0,1, \ldots, N-1$ is the angular index and the corresponding $\alpha$ is equal to $2 \pi r / N$. The intermediate signal $\hat{x}_{k}[p]$ is calculated by 
$\hat{x}_{k}[p]=v_{p}^{(k)} \sum_{n=0}^{N-1} x[n] v_{p}^{(n)}$

where $v_{p}^{(k)}$ is the kth element of $v_{p}$ and $v_{p}$ is the $p$ th column vector of $\mathbf{V}_{G}$.

It has been shown [17], [18], [23] that the DFRFT has the ability to concentrate a linear chirp into a few coefficients and that we obtain an impulselike transform analogous to what the DFT produces for a sinusoid. Fig. 4 shows the DFRFT of a complex signal containing two components: a pure $150-\mathrm{Hz}$ sinusoid and a chirp signal with a center frequency of $200 \mathrm{~Hz}$ and a chirp rate of $400 \mathrm{~Hz} / \mathrm{s}$. The frequency axis is the same as the one of the DFT. The DFRFT introduces a new angular parameter $\alpha$ to describe the linear time-frequency relation of the signal. For $\alpha=\pi / 2$, the result of the DFRFT is the same as that of the DFT. The two peaks that corresponded to the sinusoid and the chirp are well separated, which indicates that the two components have different center frequencies and chirp rates. Fig. 5 shows the 2-D view of the angle-frequency spectrum generated by the DFRFT.

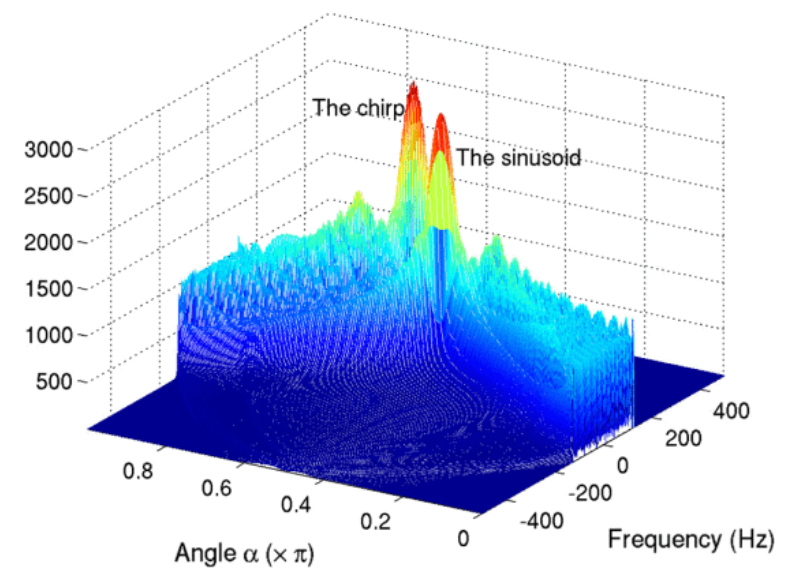

Fig. 4. Three-Dimensional Angle-Frequency Spectrum of the Signal Using the Dfrft. the Chirp Component and the Sinusoidal Component are Corresponding to Different Peaks in the Spectrum. No Cross-Term is Introduced Due to Linearity of the Dfrft.

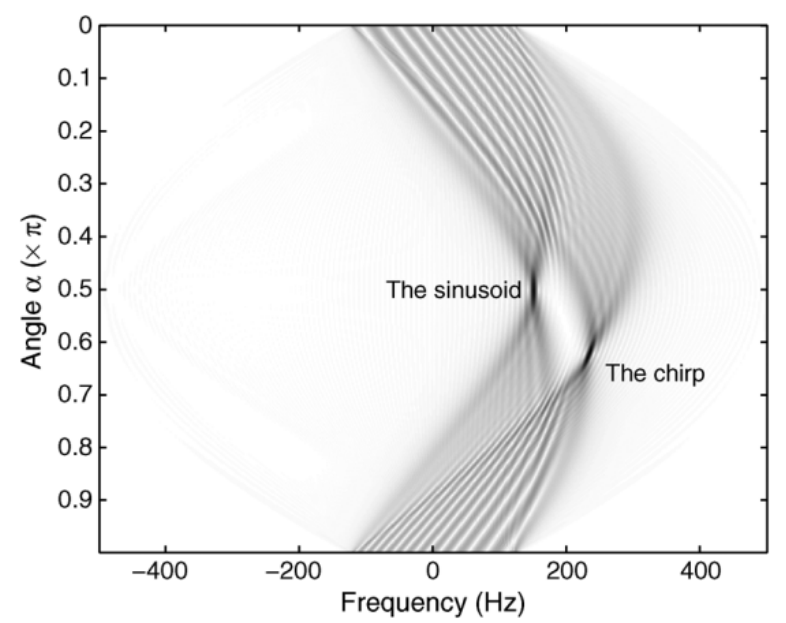

Fig. 5. Vertical View of the Angle-Frequency Spectrum Shown in Fig. 4. Black and White Correspond to the Highest and Lowest Amplitudes, Respectively.

\section{B. Vibration Estimation Method}

The Sol described in Section II-B is an example of nonstationary signals. They can be analyzed by means of using sliding short-time windows. In a short-time window starting at $m$, a second-order approximation can be applied to the vibration displacement $r_{d}$, and the Sol in (6) becomes 


$$
\begin{gathered}
x[n] \approx \sigma \exp \left[j \left(\phi-\frac{4 \pi f_{c}}{c} r_{d}[m]+\left(f_{y} \bar{y}-\frac{4 \pi f_{c} v_{d}[m]}{c f_{\mathrm{prf}}}\right) n\right.\right. \\
\left.\left.-\frac{2 \pi f_{c} a_{d}[m]}{c f_{\mathrm{prf}}^{2}} n^{2}\right)\right]+w[n], m \leq n<m+N_{w}
\end{gathered}
$$

where $N_{w}$ is the size of the window. We assume that the reflectivity of the target $\sigma$ does not change within the time window. The length of the time window is $T_{w}=f_{\mathrm{prf}} N_{w}$. When $T_{w}$ is much less than the duration of the vibration, the second-order approximation in (9) is fairly accurate. According to $\underline{(9)}, x[n]$ in a short-time window is approximately a chirp signal, and its chirp rate is linearly proportional to the instantaneous vibration acceleration $a_{d}[m]$. By estimating the chirp rates of $x[n]$ in successive sliding short-time windows, the vibrationacceleration history is estimated. The DFRFT is used to estimate the chirp rates, and the details are shown as follows.

\section{Incorporating the CZT}

Because the vibration-induced chirp rates are usually very small, a resolution enhancement algorithm, called the the chirp z-transform (CZT) algorithm, is incorporated into the DFRFT. With the CZT, a more finely spaced interpolation of the spectrum of interest can be obtained than that offered by the DFT [12], [24]. As shown in Section III-A, the final step of the DFRFT can be interpreted as the DFT of $\hat{x}_{k}[p]$ in (8) for each frequency index $k$ in (7). Therefore, we can implement the CZT algorithm in the final step of the DFRFT in order to obtain more exact peak locations with respect to angle $\alpha$ [25]. Fig. 6 shows the CZT-incorporated DFRFT of the signal with a zoom-in factor of two. The resolution of the peak position with respect to the angle $\alpha$ is improved.

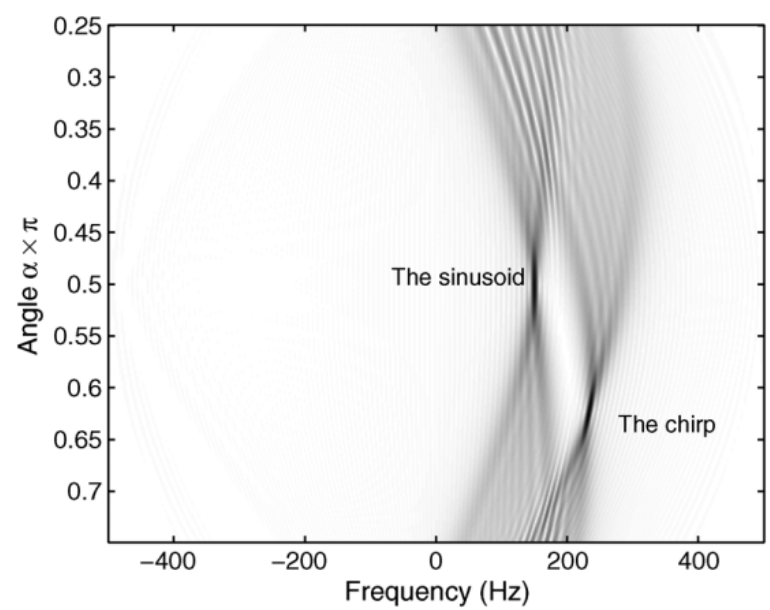

Fig. 6. CZT-Incorporated DFRFT of the Signal with a Zoom-in Factor of two. by Incorporating the CZT, the Resolution of the Peak Position with Respect to Angle $\alpha$ is Improved.

\section{Estimating Chirp Rates}

There is a one-to-one mapping from the angular position of the peak in the DFRFT plane to the chirp rate of the signal [17]. This mapping is dependent on the size of the DFRFT and the zoom-in factor of the CZT. Currently, there is no analytic form to describe the mapping. Fig. 7 shows a mapping from the peak location to the chirp rate where the DFRFT size is 160 and the zoom-in factor is 10 . The mapping is generated by first using the DFRFT to estimate signals with different chirp rates and then interpolating the estimation results with the spline function. In practice, the mapping is generated with a parameter set that works best for the particular application and is stored for later use in estimating chirp rates. Once the chirp rate is estimated by the DFRFT, the estimated vibration acceleration is calculated via 
$\hat{a}_{d}[m]=-\frac{c f_{\mathrm{prf}}^{2}}{2 \pi f_{c}} \hat{c}_{r}[m](10)$

where $\hat{c}_{r}[\mathrm{~m}]$ is the estimated chirp rate. By estimating the acceleration in sliding short-time windows, the history of the vibration acceleration is estimated. The estimated vibration spectrum can be obtained by applying the DFT to the estimated-vibration-acceleration history. The DFRFT-based vibration estimation method is summarized in Algorithm 1. Usually, the DFRFT-based method is applied to the Sol with several different window sizes to achieve the best performance.

\section{Algorithm 1 Procedure for the proposed vibration-estimation method}

1. demodulate and reformat the SAR phase history, perform autofocus;

2. apply range compression to the SAR phase history, identify the Sol;

3. choose a proper window size $N_{w}$

4. for all $m=0$ to $m=N-N_{w}+1$ do

5. apply the DFRFT to the Sol in each time window and estimate the chirp rate $\hat{c}_{r}[\mathrm{~m}]$;

6. end for

7. calculate the estimated instantaneous vibration acceleration via $\hat{a}_{d}[m]=-\left(c f_{\mathrm{prf}}^{2} / 2 \pi f_{c}\right) \hat{c_{r}}[m]$;

8. reconstruct the history of the vibration acceleration and calculate its DFT

9. spectrum;

10. repeat steps $\mathbf{2 - 8}$ for different window sizes.

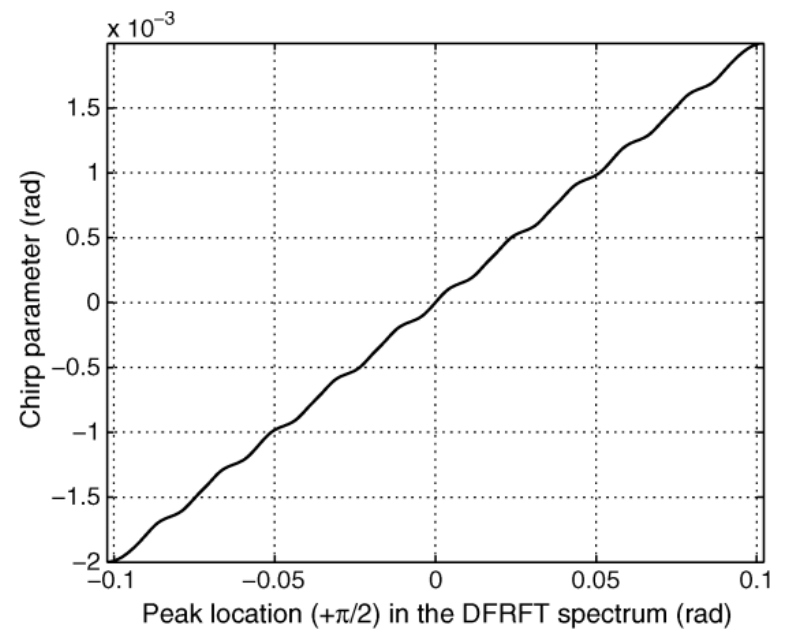

Fig. 7. One-to-One Mapping from the Peak Location in the Angle-Frequency Spectrum to the Chirp Rate Using the Dfrft. the Dfrft Size is 160, and the Zoom-in Factor is 10.

\section{SECTION IV. Performance Analysis}

In real-world applications, the performance of the proposed method is affected by the presence of noise. In the extreme case, when the Sol is highly corrupted by noise, the estimated vibration acceleration would not be reliable. Thus, we are interested in knowing the SNR threshold above which the estimation error is acceptable. To this end, we have used Monte Carlo simulations to evaluate the performance of the proposed method in estimating the chirp rate under different SNR levels. The Sol in the presence of noise in a given time window starting at $m$ can be written as

$$
s[n]=\sigma \exp \left[j\left(\phi+\omega_{c} n+c_{r} n^{2}\right)\right]+w[n](11)
$$


for $m \leq n<m+N_{w}$. The noise term $w[n]$ is modeled as a zero-mean complex-valued white Gaussian noise. The SNR is define as

$S N R=10 \log \frac{\sigma}{\sigma_{w}}(12)$

where $\sigma 2 \mathrm{w}$ is the variance of the additive noise. Because the DFRFT is evaluated on discrete angular values, the step size in angle $\alpha$ is limited to

$\rho=\frac{2 \pi}{\eta N_{D}}(13)$

where $N_{D}$ is the size of the DFRFT and $\eta$ is the zoom-in factor. This also yields a finite resolution for chirp rate estimates.

\section{A. SNR Requirements}

We have evaluated the performance of the estimator shown in Fig. 7. The estimator is used to estimate chirp rates within the range from -0.002 to $0.002 \mathrm{rad} / \mathrm{samples}^{2}$. This estimator roughly yields a resolution of $7.85 \times 10^{-5} \mathrm{rad} / \mathrm{samples}^{2}$ in estimating the chirp rate. The normalized root mean square errors (NRMSEs) for five chirp rates are plotted in Fig. 8. The five chirp rates are 0.00011, 0.00021, 0.00031, 0.00041, and 0.00051. When the SNR increases to $20 \mathrm{~dB}$, the NRMSEs of most of the chirp rates (except for $c_{r}=0.00011$ ) drop to an acceptable level (roughly 0.05). However, the errors plateau as the SNR increases. The residual errors are mainly from the quantization error due to the limited resolution. When the estimated chirp rate is on the same order of the resolution limit, a small estimation error causes large NRMSE. This is seen by observing the NRMSE of the chirp rate of 0.00011 . When the SNR is $30 \mathrm{~dB}$, the NRMSE in estimating the chirp rate of 0.00011 is still about $10 \%$.

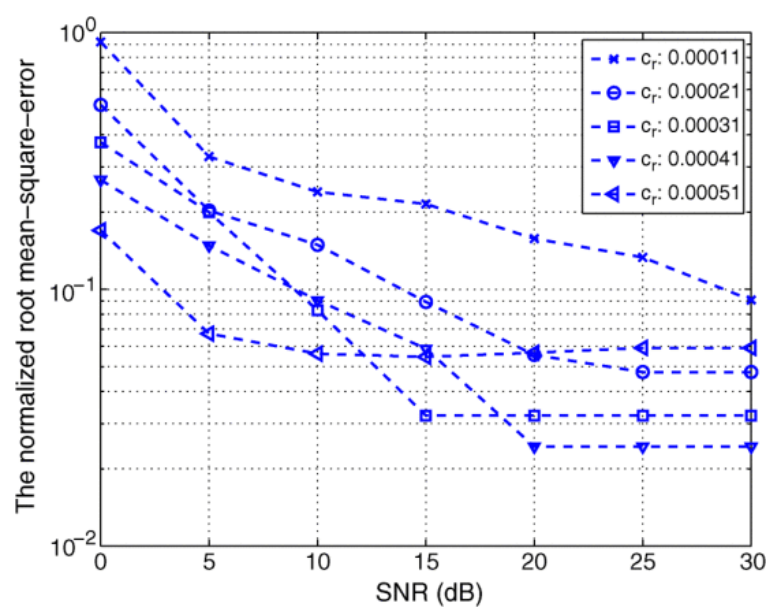

Fig. 8. Normalized Mean-Squared Error in Estimating Five Different Chirp Rates Using the Dfrft-Based Estimator Shown in Fig. 7.

Note that it is important to choose a proper setting for the estimator in terms of the DRFT size $N_{D}$ and the zoom-in factor $\eta$. The size of the DFRFT $N_{D}$ is usually determined by other factors that will be explained later in this section, and it can be larger than $N_{w}$ in some cases. By using a large zoom-in factor, the resolution of the estimator is enhanced, and the residual error is reduced. Based on the prior information of the SAR system in use, a DFRFT size and a relatively small zoom-in factor are chosen to build the first estimator. If the estimator does not fit with the chirp rates induced by the vibration, then the zoom-in factor is increased accordingly. We avoid using a very high resolution estimator (by choosing a large zoom-in factor) in the very beginning due to the following two reasons. First, if the estimator becomes too sensitive, then high-frequency noise will be 
introduced to the estimated vibration frequency. Second, the vibration-induced chirp rates may be beyond the range of the estimator.

In the remainder of this section, we assume that we work under acceptable SNRs and discuss other aspects of the performance of the proposed method.

\section{B. Resolution and Range of Estimated Vibration Frequencies}

Let us assume that the SNR requirement of the Sol is met in $N$ samples. We have $N \leq N_{I}$, where $N_{I}$ is the total number of the returned pulses in the SAR phase history. We define the effective observation time as $T=f_{\operatorname{prf}} N$. The vibration is estimated over the effective observation time. Therefore, the resolution with respect to vibration frequency is given by $1 /\left(f_{\mathrm{prf}} N\right)$ that is lower bounded by $1 /\left(f_{\mathrm{prf}} N\right)$.

Next, the maximum measurable vibration frequency (MMVF) is defined to be the maximum frequency that a SAR system can estimate without any aliasing. Theoretically, the Nyquist-Shannon sampling theorem dictates that the MMVF is upper bounded by $f_{\text {prf }} / 2$. However, such an upper bound cannot be reached using the proposed method. The length of the time window should be much less than the period of the vibration in order to reduce the error introduced by the second-order approximation. On the other hand, a certain number of samples in the time window are required to estimate the chirp rate robustly. Although it is expensive or sometimes impractical to increase the PRF, the Sol can be up sampled in order to estimate high vibration frequencies. As a remedy, we can up sample the Sol prior to applying the DFRFT to it. With up-sampling the Sol, the DFRFT size $N_{D}$ is enlarged larger than the window size $N_{w}$. According to our experience, the length of the time window has to be at least half the period of the vibration, and $N_{w}$ is at least 20 . This yields a practical MMVF that is approximately equal to $f_{\text {prf }} / 40$.

\section{MMVA and MMVD}

The minimum measurable vibration acceleration (MMVA) can be calculated from the specified parameters. When the chirp rate is small, the chirp rate can be obtained via [18]

$$
c_{r}=\frac{\pi}{N_{D}}\left(\alpha_{p}-\pi / 2\right)
$$

where $\alpha p$ is the angular position of the peak in the DFRFT spectrum. The minimum angular difference in $\alpha$ that can be differentiated by the DFRFT is $2 \pi /\left(\eta N_{D}\right)$. Therefore, the MMVA is given by

$$
a_{d}^{(\min )}=\frac{\pi c f_{\mathrm{prf}}^{2}}{\eta N_{D}^{2} f_{c}}
$$

In the special case when the vibration is a single-component harmonic oscillation, the minimum measurable vibration displacement (MMVD) can be derived in a straightforward fashion. In this case, we know that $a_{d}=$ $-4 \pi^{2} f_{v}^{2} r_{d}$, where $r_{d}$ is the vibration displacement. The vibration frequency can be estimated from the calculated DFT spectrum of the estimated vibration acceleration, and it is denoted by $f_{v}$. Therefore, the MMVD in this case is given by

$$
r_{d}^{(\min )}=\frac{c f_{\mathrm{prf}}^{2}}{2 \pi \eta N_{D}^{2} \hat{f}_{v}^{2} f_{c}}
$$

Finally, the performance limits of the proposed method are summarized in Table I.

TABLE I Performance Limits of the Dfrft-Based Sar Vibration Estimation Method 


\begin{tabular}{|l|l|}
\hline Required SNR & Roughly $20 \mathrm{~dB}$ \\
\hline Frequency resolution & $\frac{1}{f_{p r f} N} ;$ Lower-bounded by $\frac{1}{f_{p r f} N_{I}}$ \\
\hline MMVF & Theoretically $f_{p r f} / 2$; practically $f_{p r f} / 40$ \\
\hline MMVA & $\frac{\pi c f_{\text {prf }}^{2}}{\eta N_{D}^{2} f_{c}}$ \\
\hline
\end{tabular}

\begin{tabular}{r|l}
\hline \hline Parameter & \\
\hline Required SNR & roughly $20 \mathrm{~dB}$ \\
\hline Frequency resolution & $\frac{1}{f_{p r f} N}$; Lower-bounded by $\frac{1}{f_{p r f} N_{I}}$ \\
\hline MMVF & theoretically $f_{p r f} / 2 ;$ practically $f_{p r f} / 40$ \\
\hline MMVA & $\frac{\pi c f_{p r f}^{2}}{\eta N_{D}^{2} f_{c}}$ \\
\hline
\end{tabular}

TABLE II Sar System Parameters Used in the Simulation

\begin{tabular}{|l|l|}
\hline Parameter & Quantity \\
\hline Pixel dimension & $0.25 \times 0.25 \mathrm{~m} 2$ \\
\hline Patch size & $200 \times 200$ \\
\hline Patch center location & $(0,9920,-2113) \mathrm{m}$ \\
\hline Nominal resolution & $0.3 \times 0.3 \mathrm{~m} 2$ \\
\hline Carrier frequency & $f_{c}=15 \mathrm{GHz}$ \\
\hline Sent pulse bandwidth & $f_{0}=503 \mathrm{MHz}$ \\
\hline Pulse duration & $t_{c}=50 \times 10^{-3} \mathrm{~s}$ \\
\hline Length of the synthetic aperture & $L=333 \mathrm{~m}$ \\
\hline Plane velocity & $V_{a}=78 \mathrm{~m} / \mathrm{s}$ \\
\hline Sampling frequency & $3.216 \mathrm{MHz}$ \\
\hline Pulse repetition frequency & $377 \mathrm{~Hz}$ \\
\hline SNR & $20 \mathrm{~dB}$ \\
\hline
\end{tabular}

\begin{tabular}{r|l}
\hline \hline parameter & quantity \\
\hline pixel dimension & $0.25 \times 0.25 \mathrm{~m}^{2}$ \\
\hline patch size & $200 \times 200$ \\
\hline patch center location & $(0,9920,-2113) \mathrm{m}$ \\
\hline nominal resolution & $0.3 \times 0.3 \mathrm{~m}^{2}$ \\
\hline carrier frequency & $f_{c}=15 \mathrm{GHz}$ \\
\hline sent pulse bandwidth & $f_{0}=503 \mathrm{MHz}$ \\
\hline pulse duration & $t_{c}=50 \times 10^{-3} \mathrm{~s}$ \\
\hline pength of the synthetic aperture & $L=333 \mathrm{~m}$ \\
\hline plane velocity & $V_{a}=78 \mathrm{~m} / \mathrm{s}$ \\
\hline pumpling frequency & $3.216 \mathrm{MHz}$ \\
\hline SNR & $20 \mathrm{~dB}$ \\
\hline
\end{tabular}




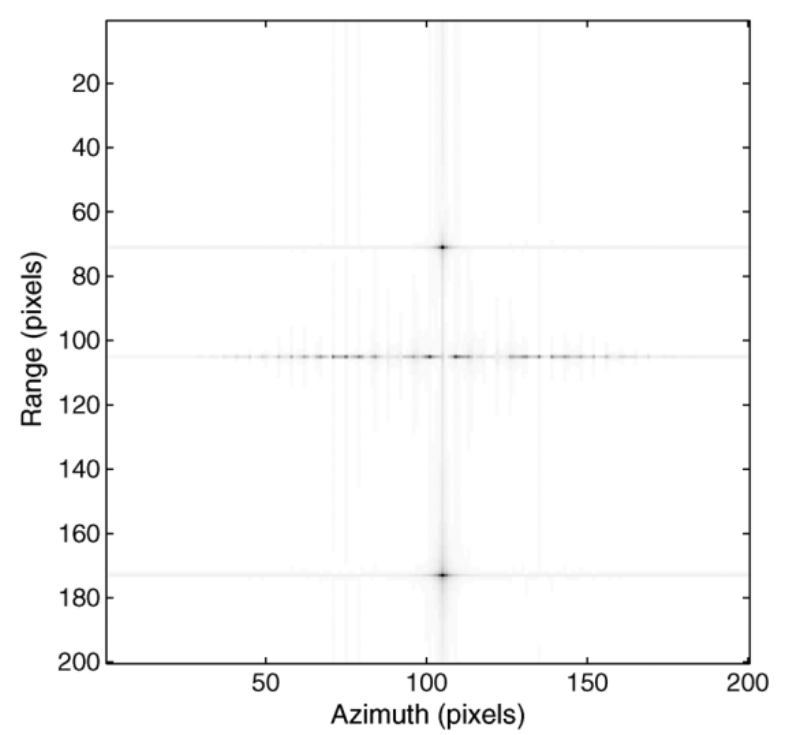

Fig. 9. Reconstructed Image from Simulated SAR Data. the Vibrating Scatterer is at the Center of the Image. note that Vibrations Introduce Many Ghost Targets in the Azimuth Direction near the Vibrating Scatterer. One Static Scatterer is Above the Vibrating Scatterer, and the Other is Below the Vibrating Scatterer.

\section{SECTION V. Simulation-Based Case Study}

A simulated example is provided to demonstrate the capability of the proposed method in estimating a multicomponent harmonic vibration under realistic SNRs (e.g., $20 \mathrm{~dB}$ ). The simulated SAR is a spotlight-mode SAR working in the $K_{u}$-band. Table II lists all the key system parameters associated with the simulation. Fig. 9 shows the reconstructed SAR image generated by using the algorithm described in [3]. There are three scatterers in the images: The one in the middle is the vibrating scatterer, and the rest are static scatterers. The vibration has two components: a 1.0- $\mathrm{Hz}$ oscillation with an amplitude of $1 \mathrm{~cm}$ and a $3.0-\mathrm{Hz}$ oscillation with an amplitude of $2 \mathrm{~mm}$. Several vibration-induced ghost targets appear around the vibration scatterer. The Sol is identified as the range line in the range-compressed phase history where the range is corresponding to that of the vibrating target. The real part of the Sol is plotted in Fig. 10. When the proposed method was applied to the simulated data, the method produced the best result when the window size $N_{w}$ was set to 20 with an upsampling factor of four. The CZT was incorporated in the DFRFT, and a zoom-in factor of eight was used. Fig. 11 shows the DFRFT of the Sol in four different time windows. Note that the positions of the peaks are slightly different from window to window, which confirms a time-varying vibration acceleration. The estimated vibration acceleration in the sliding time windows and its spectrum are shown in Figs. 12 and 13, respectively. The proposed method successfully estimated the two vibrating components with a frequency resolution of roughly $0.3 \mathrm{~Hz}$. 


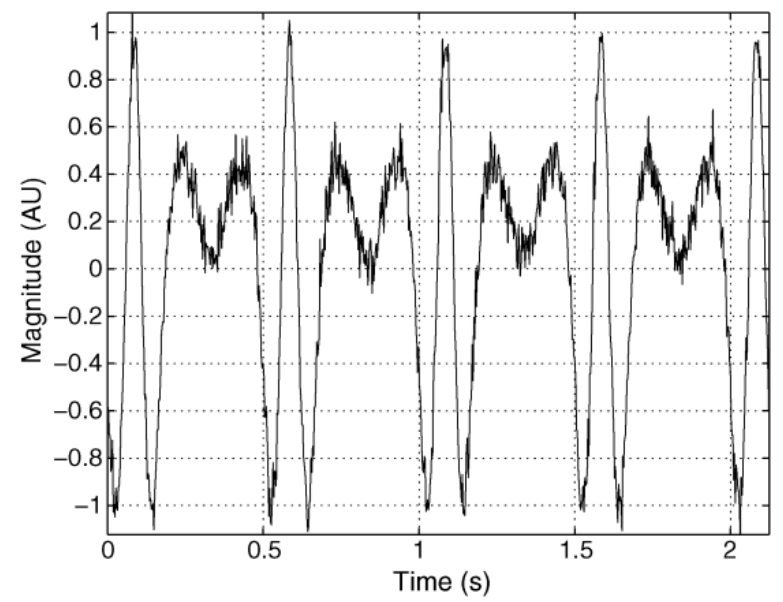

Fig. 10. Real Part of the Sol from Simulated Data. the Sol is a Nonstationary Signal, and its if is Modulated by the Vibration.
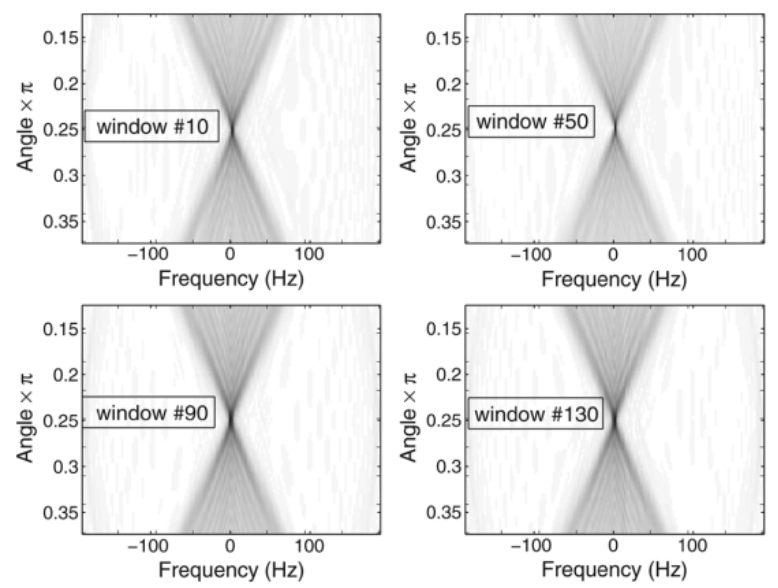

Fig. 11. DFRFT Spectra of the Sol, from Simulated Data, in Four Different Time Windows. the Peak Locations are Measured and Used to Estimate the Vibration Accelerations.

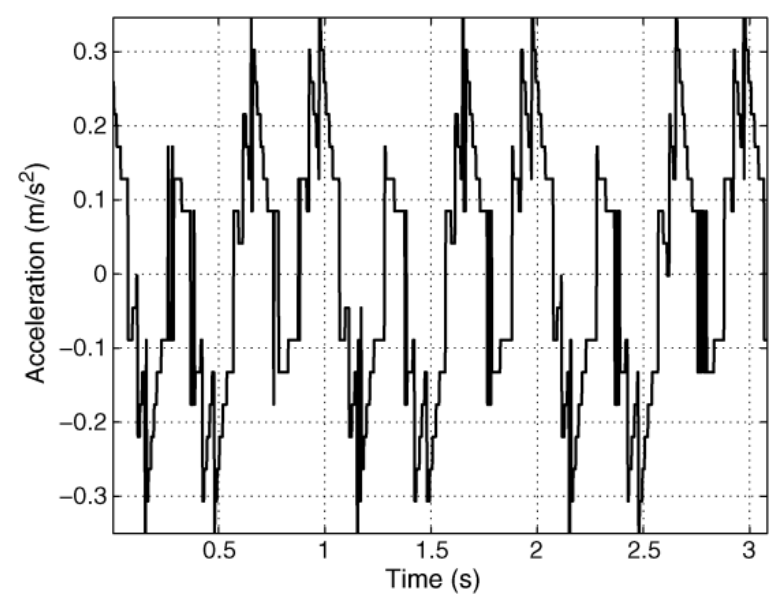

Fig. 12. Estimated Acceleration History of the Vibration from Simulated Data and by Using the DFRFT-Based Method. 


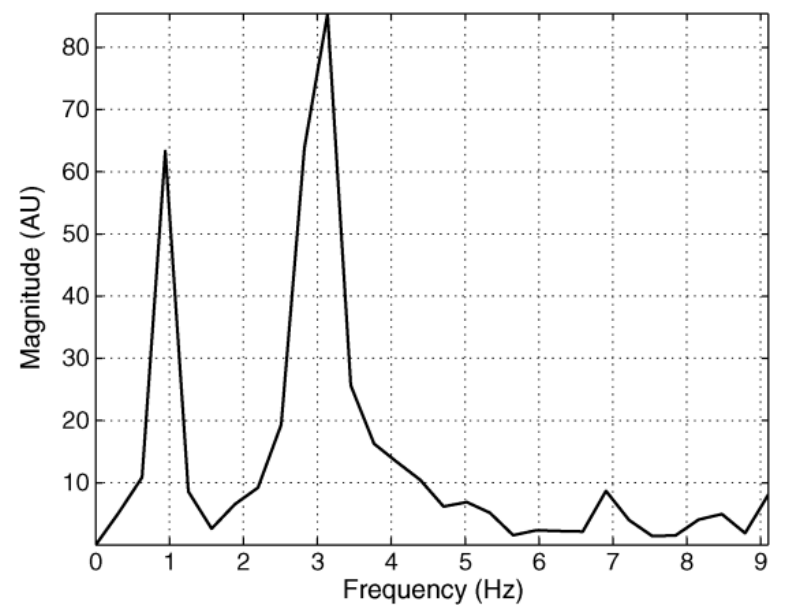

Fig. 13. Estimated Spectrum of the Vibration Using the Dfrft-Based Method Using Simulated Data. the Proposed Method Successfully Estimates Two Vibration Components: 1.0 and $3.0 \mathrm{~Hz}$.

\section{A. Comparison to a JTFA Method}

As described in Section I, the JTFA methods use time-frequency distributions to provide an analysis of the microDoppler effect. For its well-accepted performance [4], we use the smoothed pseudo Wigner-Ville distribution (SPWVD) as a representative JTFA method in the simulated example described before. To this end, we implemented the SPWVD by utilizing the widely used time-frequency toolbox [26]. The SPWVD of the Sol is shown in Fig. 14. The time-frequency representation in Fig. 14 roughly reveals 1 - and 3-Hz vibration components. However, this is only a qualitatively deduced observation. To obtain precise estimates of the instantaneous acceleration from the time-frequency representation, further estimation procedures are required. On the other hand, the proposed method provides direct quantitative estimates of the history of the vibration acceleration and the vibration frequency, and no further procedure is required.

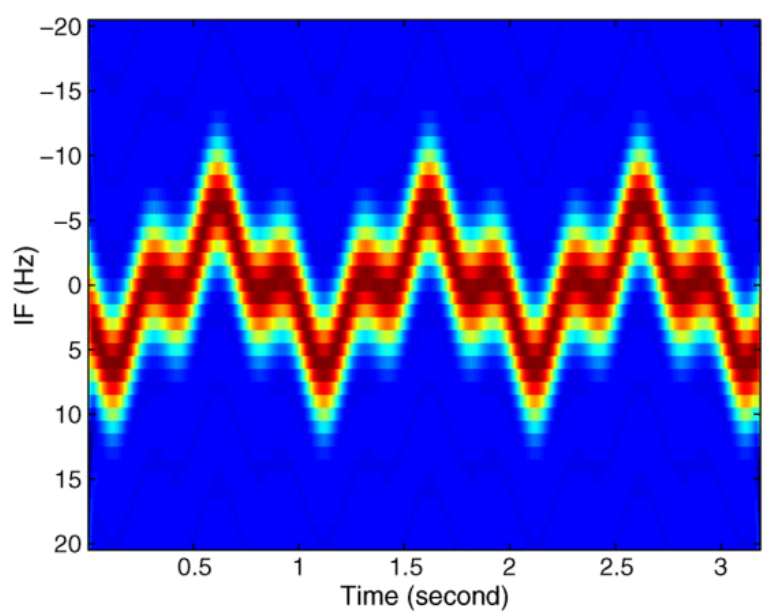

Fig. 14. Time-Frequency Representation of the Sol Using the SPWVD Approach. 


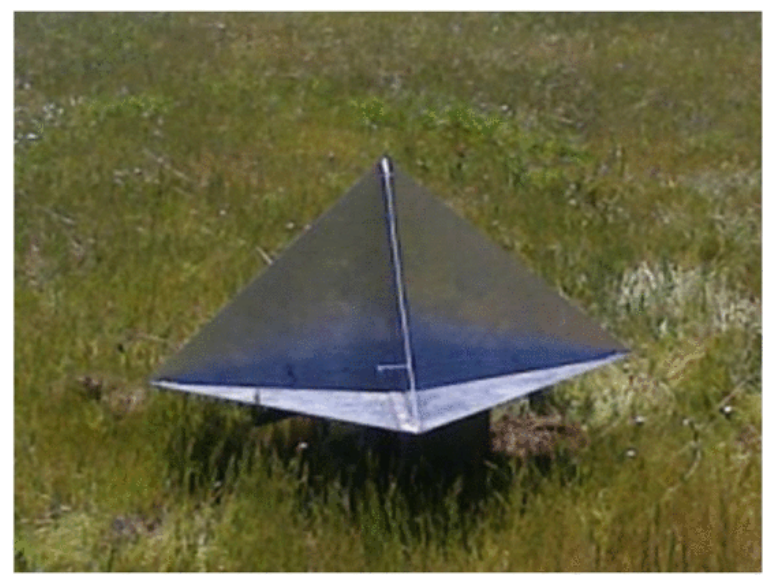

Fig. 15. Experiment I: Vibrating Target on the Test Ground near Julian, CA. the Target is an Aluminum Triangular Trihedral with a Lateral Length of $21 \mathrm{in}$. the Vibration Frequency and Amplitude were $1.0 \mathrm{~Hz}$ and $1.5 \mathrm{~cm}$, Respectively.

\section{SECTION VI. Experimental Case Studies}

Through an ongoing collaboration with GA-ASI, we conducted two experiments with the Lynx airborne $K_{u}$-band SAR system. The system parameters of the Lynx match those used in our simulations in Section V. Using flight test data from the Lynx system, the proposed method successfully estimated two vibrations from two different targets: a $1.5-\mathrm{cm} 1.0-\mathrm{Hz}$ vibration and a $1.5-\mathrm{mm} 5.0-\mathrm{Hz}$ vibration. The details of these case studies are provided next.

\section{A. Experiment I}

In the first experiment, the vibrating target was an aluminum triangular trihedral with a lateral length of 21 in, as shown in Fig. 15. The motion of the target was a single-frequency harmonic motion, driven by a dc motor attached to a crank and a piston. The vibration amplitude was $1.5 \mathrm{~cm}$, and the frequency was $1.0 \mathrm{~Hz}$. The target was positioned such that the harmonic motion is in the range direction. Fig. 16 shows the reconstructed SAR image that contains the vibrating target. The nominal resolution of the reconstructed SAR image is $0.3 \mathrm{~m}$ in each direction. The vibrating target is located at the bottom right portion of the image, and it appears as a horizontal line of the target echo and ghost targets. The vibration causes the ghost targets along the azimuth direction in the reconstructed image of the target. Note that there are also several well-separated static targets extending from the center of the image to the top right corner which are not subject to our analysis. In this experiment, the carrier frequency was $15 \mathrm{GHz}$, and the PRF was $306 \mathrm{~Hz}$. Due to seemingly limited SNR (the exact value is unknown), we selected the total observation time $T$ of this target to be $2.6 \mathrm{~s}$ centered at the time closest to target broadside. The length of each time window was 0.26 s. Fig. 17 shows the DFRFT spectra of the Sol in four different time windows. The proposed method is applied to the Sol, and the estimated-vibration-acceleration history and the corresponding vibration spectrum are shown in Figs. 18 and 19, respectively. The estimated vibration frequency is $0.9 \mathrm{~Hz}$, which is very close to the actual vibration frequency of $1.0 \mathrm{~Hz}$. 


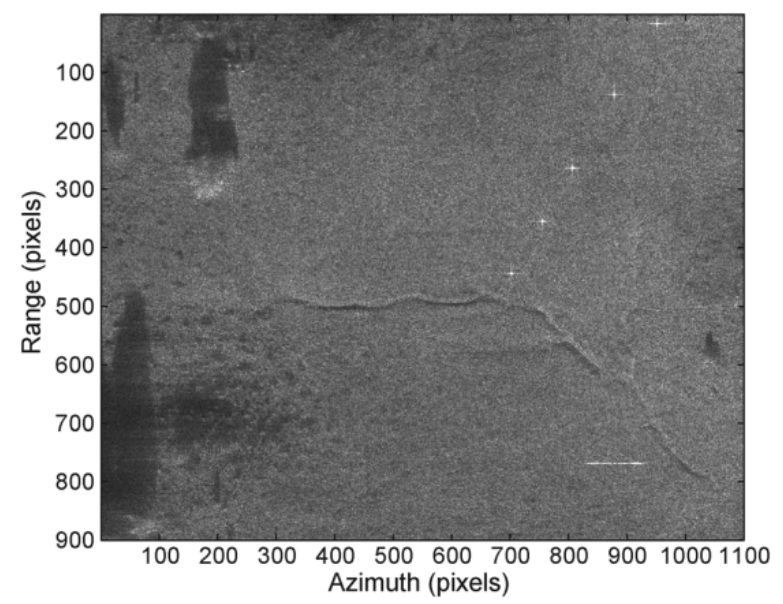

Fig. 16. Experiment I: Reconstructed SAR Image Provided by the GA-ASI Lynx System. The Vibrating Test Target is in the Lower Right Portion of This Image. There are a Few Static Targets Extending from the Center of the Image to the Top Right Corner Which are not Subject to Our Analysis.

View All
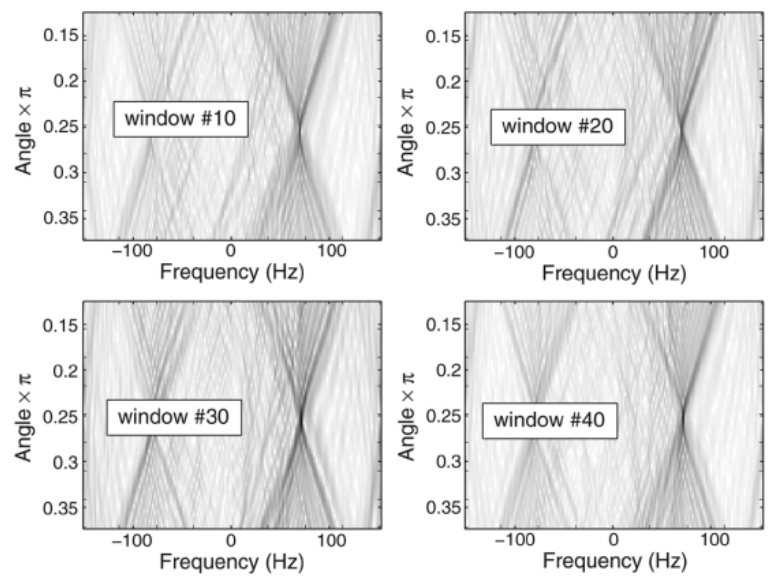

Fig. 17. Experiment I: The DFRFT Spectra of the Sol in Four Different Time Windows.

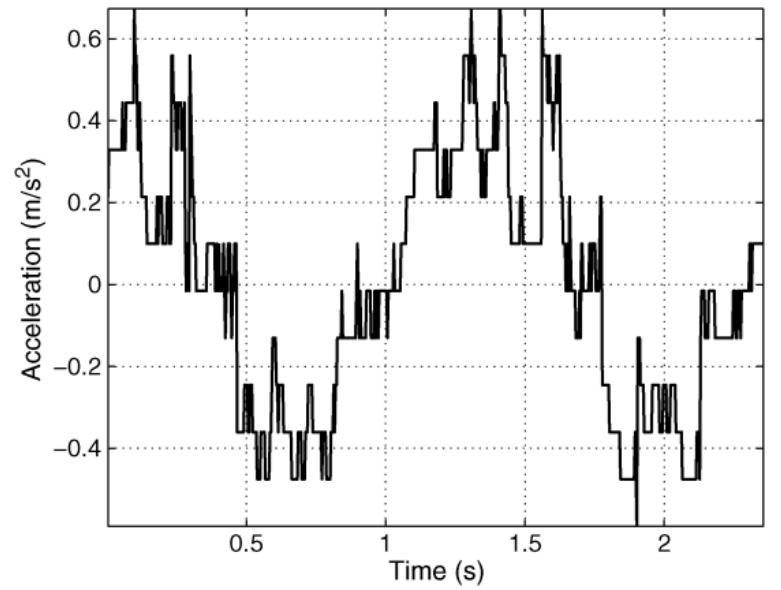

Fig. 18. Experiment I: Estimated-Vibration-Acceleration History Over $2.6 \mathrm{~s}$ Using the Proposed Method. 


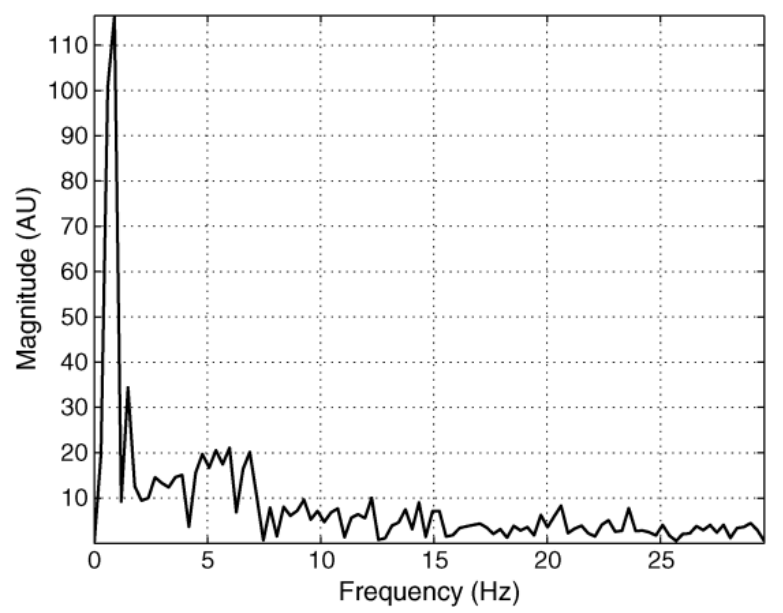

Fig. 19. Experiment I: Estimated Vibration Spectrum Using the Proposed Method. the Estimated Vibration Frequency is $0.9 \mathrm{~Hz}$; the Actual Value of the Vibration Frequency was $1 \mathrm{~Hz}$.

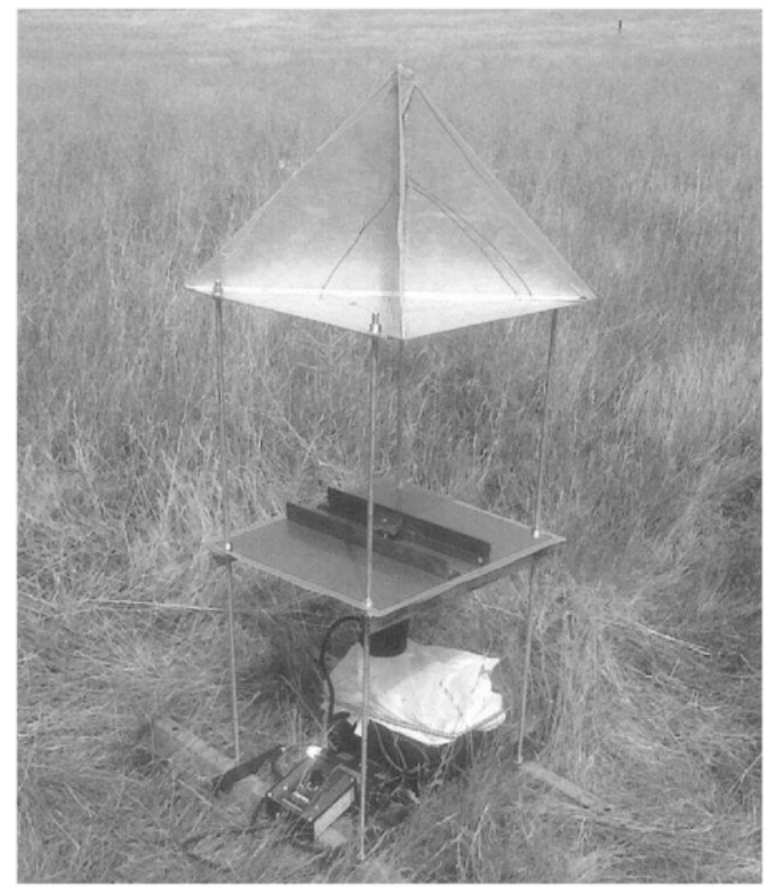

Fig. 20. Experiment II: Vibrating Target on the Test Ground Near Julian, CA. the Target is an Aluminum Triangular Trihedral with a Lateral Length of $15 \mathrm{in}$. the Actual Vibration Frequency and Amplitude were $5.0 \mathrm{~Hz}$ and $1.5 \mathrm{~mm}$, Respectively.

\section{B. Experiment II}

In the second experiment, the vibrating target was an aluminum triangular trihedral with a lateral length of 15 in, as shown in Fig. 20. Compared to the first experiment, the size of the trihedral is reduced by $40 \%$. Accordingly, the SNR was reduced substantially. In contrast to the first target that had a pure harmonic oscillation, the vibrations in the second were induced by the rotation of an unbalanced mass that was driven by a motor. The vibration's actual amplitude and frequency were $1.5 \mathrm{~mm}$ and $5 \mathrm{~Hz}$, respectively. Fig. 21 shows the SAR image that contains the vibrating target. The nominal resolution of the reconstructed SAR image is $0.3 \mathrm{~m}$ in each direction. The vibrating target is at the bottom right portion of the image. The region near the vibrating target is magnified and displayed in the inset below the SAR image. Several ghost targets appear along the azimuth direction. In this experiment, the carrier frequency was $15 \mathrm{GHz}$, and the PRF was $270 \mathrm{~Hz}$. Due to limited 
SNR, we selected the total observation time of this target to be $1 \mathrm{~s}$, centered at the time closest to target broadside. The length of each time window was chosen to be $0.1 \mathrm{~s}$.

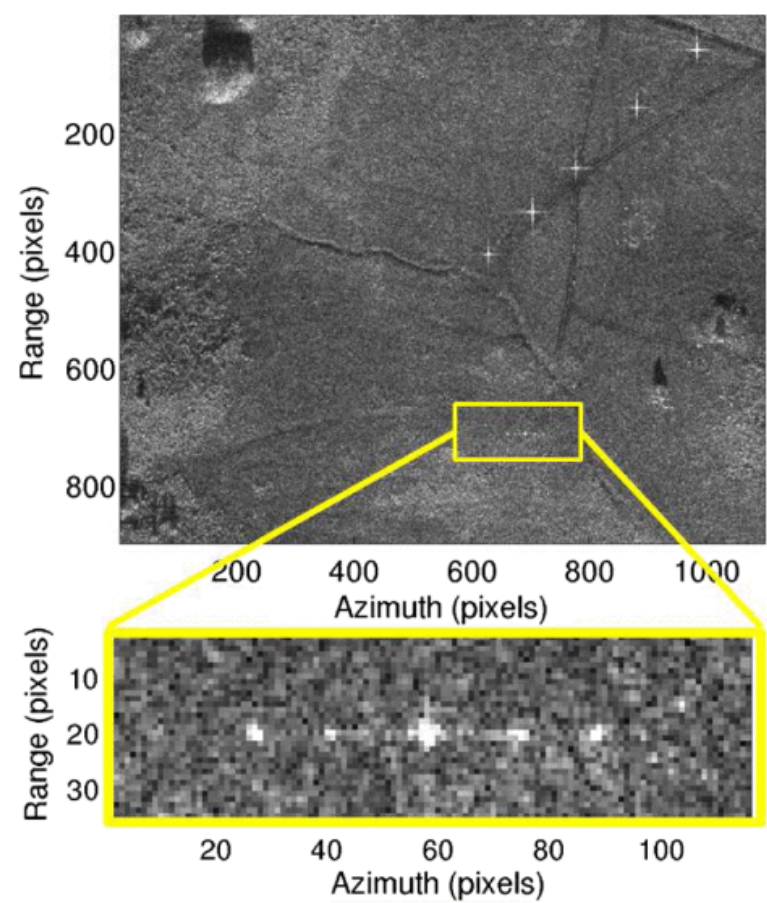

Fig. 21. Experiment II: the Reconstructed SAR Image Provided by the GA-ASI Lynx System. the Vibrating Target is in the Lower Right Portion of This Image.

Fig. 22 shows the DFRFT spectra of the Sol in four different time windows. The proposed method was applied to the Sol, and the estimated-vibration-acceleration history and the corresponding vibration spectrum are shown in Figs. 23 and 24, respectively. The estimated vibration frequency is $5.2 \mathrm{~Hz}$, which is close to the actual vibration frequency of $5.0 \mathrm{~Hz}$.
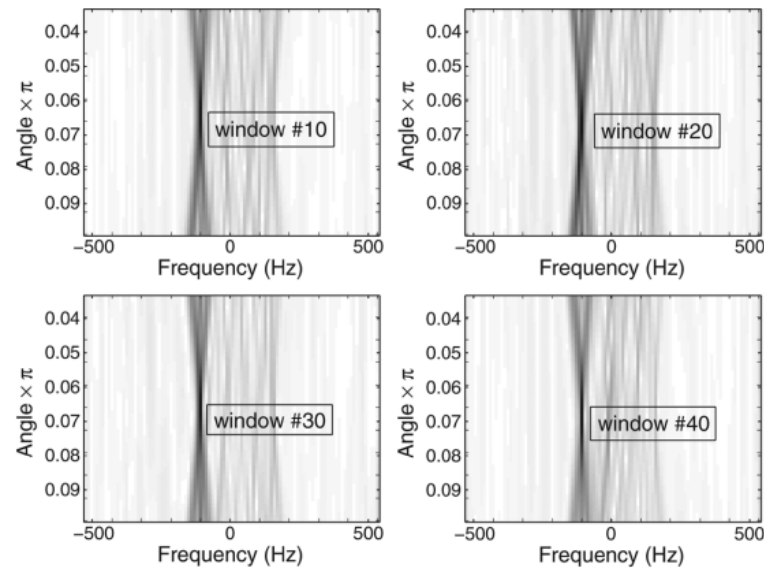

Fig. 22. Experiment II: Dfrft Spectra of the Sol in Four Different Time Windows. 


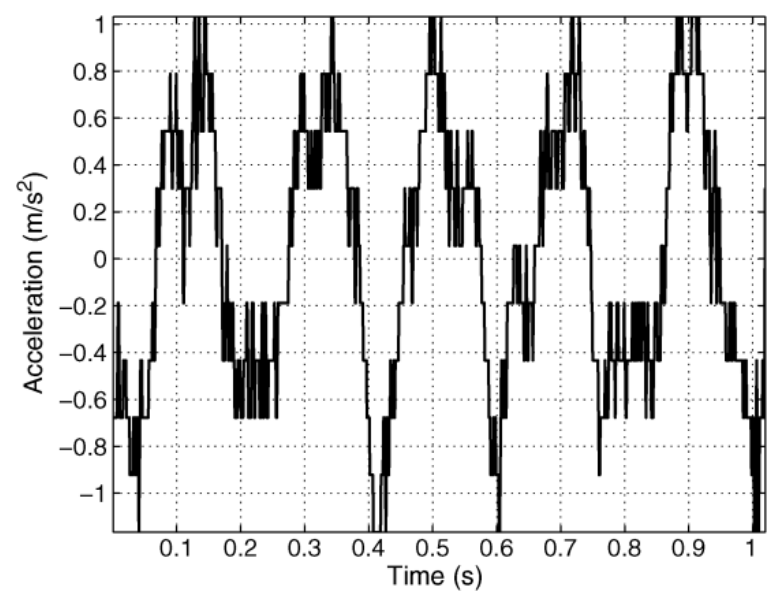

Fig. 23. Experiment II: Estimated-Vibration-Acceleration History Over $1 \mathrm{~s}$ Using the Proposed Method.

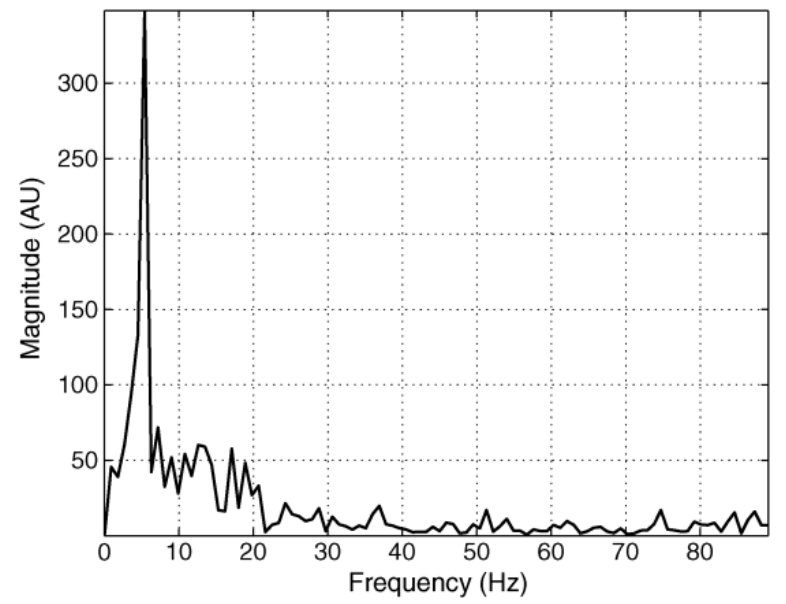

Fig. 24. Experiment II: Estimated Vibration Spectrum Using the Proposed Method. the Estimated Vibration Frequency is $5.2 \mathrm{~Hz}$; the Actual Value was $5 \mathrm{~Hz}$.

\section{SECTION VII. Conclusion}

In this paper, a DFRFT-based method has been devised to estimate low-level vibrations of ground targets using the SAR platform. Unlike the JTFA, which merely provides a qualitative illustration of the micro-Doppler effect, the proposed method provides quantitative estimates of the vibration signature directly from the SAR phase history, thereby producing the history of the instantaneous acceleration and the spectrum of the vibration. The performance of the proposed method has been characterized quantitatively in terms of measurable vibration frequency and displacement, as well as the signals' SNR (in the SAR phase history) and observation window. In experiments utilizing the GA-ASI Lynx system, the proposed method successfully retrieved two low-level vibrations from two different targets (with different SNRs).

The proposed SAR-based vibration estimation method adds a new capability to modern SAR imaging systems. As such, it enhances the diversity and utility of SAR in applications such as target feature extraction and object recognition and classification. In our future work, the effects of clutter and multiple scatterers will be carefully examined. Modeling of real-world vibrating objects, such as buildings and vehicles, could also enable a better understanding of the micro-Doppler effects in the SAR phase history and could improve the performance of the proposed method in specific applications.

\section{ACKNOWLEDGEMENT}

The authors would like to thank GA-ASI (San Diego, CA) for making the Lynx system available to this project. 


\section{References}

1. I. G. Cumming, F. H. Wong, Digital Processing of Synthetic Aperture Radar Data: Algorithms and Implementation, MA, Norwood:Artech House, 2005.

2. M. Soumekh, Synthetic Aperture Radar Signal Processing With MATLAB Algorithms, New York:Wiley, 1999.

3. C. V. Jakowatz, D. E. Wahl, P. H. Eichel, D. C. Ghiglia, P. A. Thompson, Spotlight-Mode Synthetic Aperture Radar: A Signal Processing Approach, New York:Springer-Verlag, 1996.

4. V. C. Chen, F. Li, S.-S. Ho, H. Wechsler, "Micro-Doppler effect in radar: Phenomenon model and simulation study", IEEE Trans. Aerosp. Electron. Syst., vol. 42, no. 1, pp. 2-21, Jan. 2006.

5. M. Regg, E. Meier, D. Nesch, "Vibration and rotation in millimeter-wave SAR", IEEE Trans. Geosci. Remote Sens., vol. 45, no. 2, pp. 293-304, Feb. 2007.

6. Q. Wang, M. Xing, G. Lu, Z. Bao, "High-resolution three-dimension radar imaging for rapidly spinning target", IEEE Trans. Geosci. Remote Sens., vol. 46, no. 1, pp. 22-30, Jan. 2008.

7. X. Bai, M. Xing, F. Zhou, G. Lu, Z. Bao, "Imaging of micromotion targets with rotating parts based on empiricalmode decomposition", IEEE Trans. Geosci. Remote Sens., vol. 46, no. 11, pp. 3514-3523, Nov. 2008.

8. X. Li, B. Deng, Y. Qin, H. Wang, Y. Li, "The influence of target micromotion on SAR and GMTI", IEEE Trans. Geosci. Remote Sens., vol. 49, no. 7, pp. 2738-2751, Jul. 2011.

9. Q. Wang, M. Pepin, R. J. Beach, R. Dunkel, T. Atwood, A. W. Doerry, B. Santhanam, W. Gerstle, M. M. Hayat, "Demonstration of target vibration estimation in synthetic aperture radar imagery", Proc. IEEE Int. Geosci. Remote Sens. Symp., pp. 4083-4086, 2011.

10. R. K. Raney, "Synthetic aperture imaging radar and moving targets", IEEE Trans. Aerosp. Electron. Syst., vol. AES-7, no. 3, pp. 499-505, May 1971.

11. T. Sparr, B. Krane, "Micro-Doppler analysis of vibrating targets in SAR", Proc. Inst. Elect. Eng. Radar Sonar Navigat., vol. 150, no. 4, pp. 277-283, Aug. 2003.

12. A. V. Oppenheim, R. W. Schafer, J. R. Buck, Discrete-Time Signal Processing, NJ, Upper Saddle River:PrenticeHall, 2002.

13. L. Cohen, TimeFrequency Transforms for Radar Imaging and Signal Analysis, NJ, Upper Saddle River:PrenticeHall, 1995.

14. S. Qian, D. Chen, Introduction to Joint TimeFrequency AnalysisMethods and Applications, NJ, Englewood Cliffs:Prentice-Hall, 1996.

15. S. I. Tsunoda, F. Pace, J. Stence, M. Woodring, W. H. Hensley, A. W. Doerry, B. C. Walker, "Lynx: A highresolution synthetic aperture radar", Proc. SPIERadar Sensor Technol. IV, vol. 3704, pp. 20, 1999.

16. Q. Wang, M. Pepin, B. Santhanam, T. Atwood, M. M. Hayat, "SAR-based vibration retrieval using the fractional Fourier transform in slow time", Proc. SPIERadar Sensor Technol. XIV, vol. 7669, pp. 766911-1766911-10, 2010.

17. J. G. Vargas-Rubio, B. Santhanam, "On the multiangle centered discrete fractional Fourier transform", IEEE Signal Process. Lett., vol. 12, no. 4, pp. 273-276, Apr. 2005.

18. J. G. Vargas-Rubio, B. Santhanam, "The centered discrete fractional Fourier transform and linear chirp signals", Proc. 11th DSP Workshop, pp. 163-167, 2004.

19. V. Namias, "The fractional order Fourier transform and its applications to quantum mechanics", IMA J. Appl. Math, vol. 25, no. 3, pp. 241-265, 1980.

20. L. B. Almeida, "The fractional Fourier transform and time-frequency representations", IEEE Trans. Signal Process., vol. 42, no. 11, pp. 3084-3091, Nov. 1994.

21. B. Santhanam, J. H. McClellan, "The discrete rotational Fourier transform", IEEE Trans. Signal Process., vol. 44, no. 4, pp. 994-998, Apr. 1996.

22. S. C. Pei, J. J. Ding, "Relations between fractional operations and time-frequency distributions and their applications", IEEE Trans. Signal Process., vol. 49, no. 8, pp. 1638-1655, Aug. 2001.

23. J. G. Vargas-Rubio, B. Santhanam, "An improved spectrogram using the multiangle centered discrete fractional Fourier transform", Proc. ICASSP, vol. 4, pp. 505-508, 2005.

24. L. R. Rabiner, R. W. Schafer, C. M. Rader, "The chirp $\backslash$ \$Z\\$-transform algorithm", IEEE Trans. Audio Electroacoust., vol. AU-17, no. 2, pp. 86-92, Jun. 1969. 
25. L. S. Reddy, B. Santhanam, M. M. Hayat, "Multicomponent chirp demodulation using discrete fractional Fourier transform", Proc. 12th Digital Signal Process. Workshop, pp. 418-422, 2006.

26. F. Auger, O. Lemoine, P. Goncalvs, P. Flandrin, The TimeFrequency Toolbox, pp. 1995-1996, Oct. 2005. Keywords

INSPEC: Controlled Indexing

discrete Fourier transforms,

$\underline{\text { radar signal processing, }}$

$\underline{\text { synthetic aperture radar, }}$

vibrations

INSPEC: Non-Controlled Indexing

$\underline{S A R}$,

vibration estimation,

discrete fractional Fourier transform,

synthetic aperture radar,

DFRFT,

phase modulation,

time-varying acceleration,

quasiinstantaneous chirp rate,

vibration frequency,

two-component vibration,

signal-to-noise level,

frequency $1 \mathrm{~Hz}$,

frequency $5 \mathrm{~Hz}$

Author Keywords

Fractional Fourier transform,

joint time-frequency analysis (JTFA),

$\underline{\text { micro-Doppler effect, }}$

subaperture,

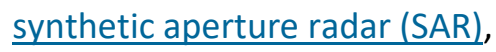

vibration 